\title{
Optical properties of germanium dioxide in the rutile structure
}

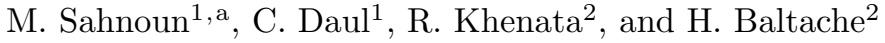 \\ 1 Département de Chimie, Université de Fribourg, Chemin du Musée 09, 1700 Fribourg, Switzerland \\ 2 Departement de Technologie, Université de Mascara, Route de Mamounia, 22000 Mascara, Algeria
}

Received 22 February 2005

Published online 13 July 2005 - (c) EDP Sciences, Società Italiana di Fisica, Springer-Verlag 2005

\begin{abstract}
We present first-principles calculations for the optical properties of germanium dioxide in the rutile structure. The electronic band structure has been calculated self-consistently within the local density approximation using the full-potential linearized augmented plane wave method. The electronic band structure shows that the fundamental energy gap is direct at the center of the Brillouin zone. The determinant role of a band structure computation with respect to the analysis of the optical properties is discussed.
\end{abstract}

PACS. 71.15.Ap Basis sets (LCAO, plane-wave, APW, etc.) and related methodology (scattering methods, ASA, linearized methods, etc.) - 74.25.Jb Electronic structure - 74.25.Gz Optical properties

\section{Introduction}

Germanium dioxide is particulary interesting to study because it is similar to silicon dioxide in many respects. $\mathrm{GeO}_{2}$ occurs in three stable forms at ambient temperature [1]. The first form has the rutile tetragonal structure $\left(\mathrm{P} 4_{2} / \mathrm{mnm}\right)$ with octahedral coordination [2]. The second form has the trigonal structure of $\alpha$-quartz [3], with tetrahedral coordination. The third form is glassy, having the same short-range correlation [4]. The tetragonal and trigonal crystalline forms differ substantially in their chemical properties, especially solubility, in their densities and indices of refraction. Over the past three decades various physical and thermodynamical properties of rutiletype $\mathrm{GeO}_{2}$ have been investigated: elastic properties [5], compressibility [6], entropy and enthalpy [7], thermal expansion [8], structure [9], melting phenomena [10], electronic properties [11-16] and high-pressure behavior [18].

The response function for the optical properties of a solid, describing the absorption or emission of electrons or photons, is the dielectric function. One may calculate the dielectric function from accurate band structure calculations, thereby allowing one to assign the spectral features to specific excitations within the Brillouin zone, as well as obtaining interband topologies. However, to our knowledge, up to now, there has been no calculation of the optical properties of the rutile-type $\mathrm{GeO}_{2}$ that includes the full transition matrix. The determination of optical properties requires, apart from the Kohn-Sham eigenvalues, the explicit use of the wave functions which should be calculated as accurately as possible. The aim of this paper is

\footnotetext{
a e-mail: mohammed.sahnoun@unifr.ch
}

to present the results of a theoretical investigation of the electronic structure and optical spectra of $\mathrm{GeO}_{2}$ which crystallizes in a rutile-type lattice.

The organization of the paper is as follows: we explain the computational method in Section 2. The results are presented and discussed in Section 3 for the electronic structure and the optical properties. A brief conclusion is drawn in Section 4.

\section{Computational method}

$\mathrm{GeO}_{2}$ crystallizes with the bulk rutile structure (space group $\mathrm{P} 4_{2} / \mathrm{mnm}$ ) [2,19,20]. In Figure 1 a schematic representation of the lattice is depicted. The atoms coordinates are Ge: $(0,0,0),\left(\frac{1}{2}, \frac{1}{2}, \frac{1}{2}\right)$ and $\mathrm{O}$ : $\pm(x, x, 0), \pm\left(\frac{1}{2}+x, \frac{1}{2}-x, \frac{1}{2}\right)$. The structure consists of chains of $\mathrm{GeO}_{6}$ octahedra and each pair shares opposite edges. Each Ge atom is surrounded octahedrally by six oxygen atoms, whereas each oxygen is surrounded by three Ge atoms arranged as corners of an equilateral triangle. The structure has a 6:3 coordination. Each octahedron is not regular, showing a slight orthorhombic distortion and is in contact with ten neighbor octahedra, two sharing edge oxygen pairs and eight sharing corner oxygen ions [21].

The geometry of rutile-type structure of $\mathrm{GeO}_{2}$ has been optimized by varying the positional parameter and cell length, and calculating the total energy, until a minimum has been found, using the first-principles FP-LAPW method in a recent implementation [22-25]. This code uses a basis set obtained by dividing the unit cell into non-overlapping atomic spheres (centered at the 


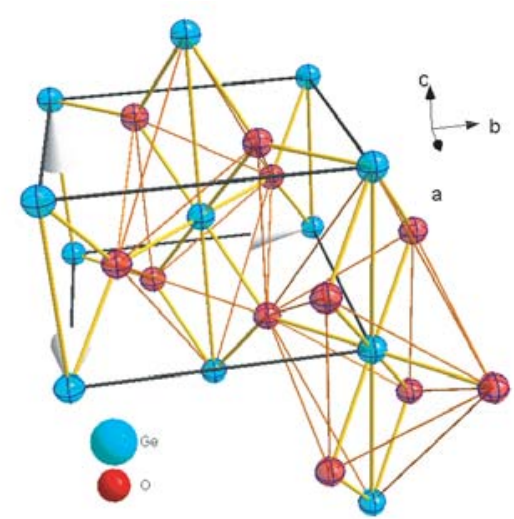

Fig. 1. Rutile structure, blue spheres represent germanium atoms and red spheres the oxygen atoms.

atomic sites) and an interstitial region. In the atomic sphere, a linear combination of radial functions times spherical harmonics is used and in the interstitial region, a plane wave expansion is augmented by an atomiclike function in every atomic sphere. This approach has been extensively tested and is among the most accurate methods for performing electronic structure calculations for crystals. The exchange and correlation potential within the Local Density Approximation (LDA) is calculated using the scheme of Hedin-Lundqvist [26]. The sphere radii of $\mathrm{Ge}$ and $\mathrm{O}$ have been chosen as 1.8 and 1.6 atomic units (a.u.), respectively. Within these spheres, the charge density and potential are expanded in terms of crystal harmonics up to an angular momentum $l=10$. For the calculation of the optical properties, a dense mesh of uniformly distributed $k$-points is required. Hence, the Brillouin zone integration was performed using the tetrahedron method with $105 k$-points in the irreducible part of the Brillouin zone without broadening. Well converged solutions are obtained for $\mathrm{R}_{M T} \cdot \mathrm{K}_{\max }=7$, $\mathrm{R}_{M T}$ labelling the atomic sphere radii, and $\mathrm{K}_{\max }$ the interstitial plane wave cut-off. The optical properties have been obtained through the frequency-dependent dielectric function, $\varepsilon(\omega)=\varepsilon_{1}(\omega)+i \varepsilon_{2}(\omega)$, using the formalism of Ehrenreich and Cohen [27]. The imaginary part of the dielectric function is given as

$$
\varepsilon_{2}(\omega)=\frac{e^{2} \hbar}{\pi m^{2} \omega^{2}} \sum_{v, c} \int_{B Z}\left|M_{c v}(\mathbf{k})\right|^{2} \delta\left[\omega_{c v}(\mathbf{k})-\omega\right] d^{3} \mathbf{k}
$$

where the integral is over the first Brillouin zone, $M_{c v}(\mathbf{k})=\left\langle u_{c \mathbf{k}}|\widehat{\mathbf{e}} \cdot \nabla| u_{v \mathbf{k}}\right\rangle$ are the dipole matrix elements for direct transitions between valence- and conductionband states, $\hbar \omega_{c v}(\mathbf{k})=E_{c \mathbf{k}}-E_{v \mathbf{k}}$ is the excitation energy, $\widehat{\mathbf{e}}$ is the polarization vector of the electric field, and $u_{c \mathbf{k}}(\mathbf{r})$ is the periodic part of the Bloch wave function for a conduction-band state with wave vector $\mathbf{k}$. Excitation energies between the valence-band and conductionband states up to approximately $19 \mathrm{eV}$ from the valenceband edge were considered. Brillouin-zone integration has been performed within the linear analytic tetrahedron scheme [28].
Table 1. Calculated structural parameters of $\mathrm{GeO}_{2}$. Lattice constants are in $\AA$.

\begin{tabular}{llllll}
\hline $\begin{array}{l}\text { Lattice } \\
\text { constants }\end{array}$ & Present & \multicolumn{2}{c}{ Experiments } & \multicolumn{2}{c}{ Calculations } \\
& & {$[19]$} & {$[20]$} & {$[29]$} & {$[30]$} \\
\hline$a$ & 4.3897 & 4.4066 & 4.4066 & 4.3838 & 4.3515 \\
$c$ & 2.8740 & 2.8619 & 2.8619 & 2.8637 & 2.8628 \\
$x$ & 0.3070 & 0.3059 & 0.3060 & 0.3059 & 0.3060 \\
\hline
\end{tabular}

Table 2. Critical point energies (eV) for polarization perpendicular $\left(E_{\perp}\right)$ and parallel $\left(E_{\|}\right)$to the $c$ axis. The notation of the optical peaks is arbitrary.

\begin{tabular}{rrrr}
\hline$E_{\perp}$ & Energy & $E_{\|}$ & Energy \\
\hline $\mathrm{A}_{1}$ & 6.38 & $\mathrm{X}$ & 7.37 \\
$\mathrm{~A}_{2}$ & 8.12 & & \\
$\mathrm{~B}_{1}$ & 10.42 & $\mathrm{Y}_{1}$ & 8.62 \\
$\mathrm{~B}_{2}$ & 11.23 & $\mathrm{Y}_{2}$ & 9.60 \\
$\mathrm{~B}_{3}$ & 12.52 & $\mathrm{Y}_{3}$ & 10.29 \\
& & $\mathrm{Y}_{4}$ & 11.04 \\
$\mathrm{C}_{1}$ & 14.48 & $\mathrm{Z}_{1}$ & 13.62 \\
$\mathrm{C}_{2}$ & 15.42 & $\mathrm{Z}_{2}$ & 14.36 \\
& & $\mathrm{Z}_{3}$ & 15.42 \\
\hline
\end{tabular}

\section{Results and discussions}

The calculated structural parameters of the rutile $\mathrm{GeO}_{2}$ are given in Table 1. They have been obtained as a result of the minimization of the lattice ground-state energies. The obtained results are compared with available experimental data and other calculations. A good agreement within a $1 \%$ interval is found. In Figure 2, we show the band structure for $\mathrm{GeO}_{2}$. The tightly bound $\mathrm{O} 2 s$ is the lowest bands and it has $1.8 \mathrm{eV}$ of bandwidth. The upper valence bands with a width of $10.49 \mathrm{eV}$, are composed mainly of $\mathrm{O} 2 p$ states. A $1.80 \mathrm{eV}$ direct-forbidden gap occurs at $\Gamma$ and is expected to be lower than the experimental value. The fundamental absorption edge has been studied by Stapelbroek and Evans [17]. The fundamental gap was concluded to be direct and optically-forbidden of $4.68 \mathrm{eV}$ magnitude. Christie and Chelikowsky obtained a value of $2.4 \mathrm{eV}$ by using a pseudopotential density functional method [12]. The lower conduction-band states, with a full width of $11.10 \mathrm{eV}$, are composed primarily of $\mathrm{O} 2 p$ and Ge $s-p$ states. The imaginary part of the dielectric function for both $\mathbf{E}_{\perp}$ and $\mathbf{E}_{\|}$polarizations are shown in Figure 3. The obtained interband transition energies are indicated with arrows and are listed in Table 2. LDA underestimates the excitation energies as evident by the shift in the fundamental absorption edge to lower energies.

The fundamental absorption edge for $\mathbf{E}_{\perp}$ occurs at $\sim 1.80 \mathrm{eV}$, resulting from transitions between the topmost valence band and the bottom of the conduction band along the $\Gamma$ point. The two prominent features, designated as $A_{1}$ and $A_{2}$ in Figure 3 , are attributed to splitting in the $\mathrm{O} 2 p_{x, y}$ orbitals. The first major peak, which we attribute to $A_{1}$, occurs at $6.38 \mathrm{eV}$ and results 


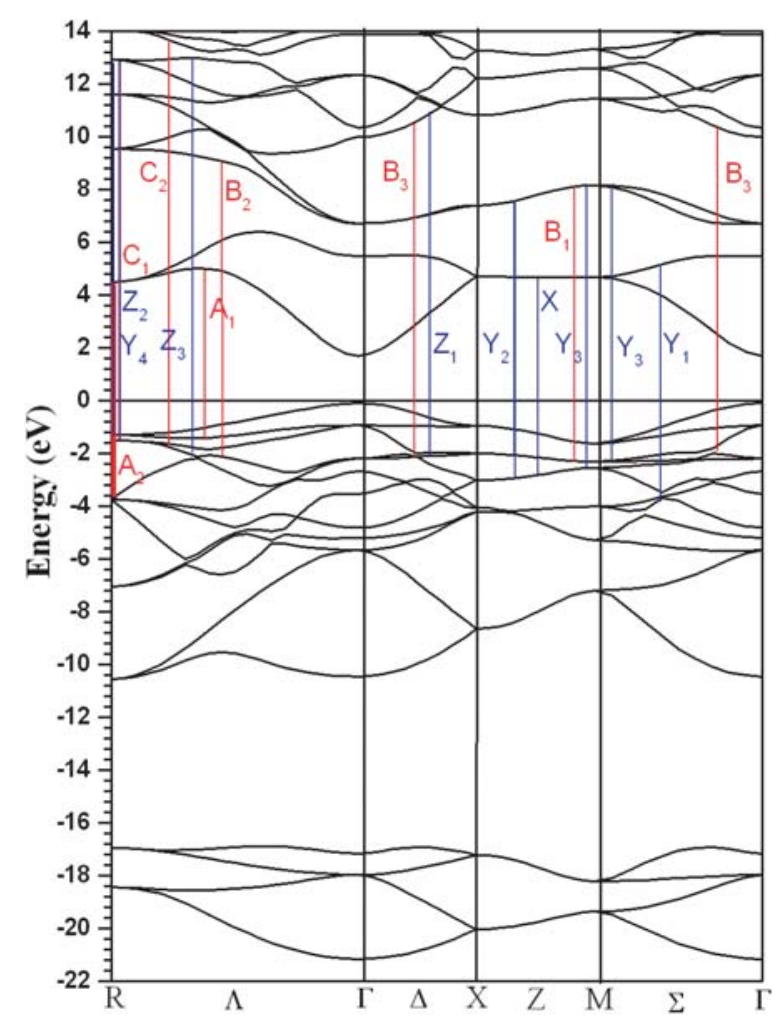

Fig. 2. Band structure of $\mathrm{GeO}_{2}$ along the high-symmetry directions of the irreductible Brillouin zone. The valence-band maximum is taken as the zero of energy.

from transitions between valence band 15 to the first conduction band along the $\mathrm{R}-\Gamma$ direction (we label the valence bands from 1-16 and the conduction bands from 1 upwards). The second peak, which we assign to $A_{2}$, occurs at $8.12 \mathrm{eV}$ and results from transitions between valence bands 11 and 12 to the two first conduction bands along the $\mathrm{R}$ point. The next three features, which we attribute to $B_{1}, B_{2}$ and $B_{3}$ occur at $10.42,11.23$ and $12.52 \mathrm{eV}$, respectively; the first one results from transitions between valence band 14 to the third conduction band along the $\mathrm{X}-\mathrm{M}$ direction, the second one results from transitions between valence band 13 to the third conduction band along $\Lambda$ direction. And the third peak results from transitions between valence bands 14 and 15 to the conduction bands 5 and 6 along $\mathrm{M}-\Gamma$ and $\Delta$ directions, respectively. The next high-energy structures are associated with $C_{1}$ and $C_{2}$ which occur at 14.48 and $15.42 \mathrm{eV}$, and result from transitions between valence bands 14 and 13 to the conduction bands 8 and 9 in the neighborhood of $\mathrm{R}$ and along $\mathrm{M}-\Gamma$, respectively.

The absorption edge for $\mathbf{E}_{\|}$polarization occurs at $\sim 1.80 \mathrm{eV}$ resulting from transition between the top of the valence band and the bottom of the conduction band with the majority of the structure occurring on the $\Delta-\mathrm{X}-\mathrm{M}-\Sigma$ plane. As seen in Figure 3, one prominent feature at $10.29 \mathrm{eV}$, labelled $B_{3}$, is obtained for $\mathbf{E}_{\|}$and is attributed to transitions from the $\mathrm{O} 2 p_{z}$ orbitals. The first major peak for $\mathbf{E}_{\|}$polarization occurs at $7.37 \mathrm{eV}$ and results from transitions between valence band 12 to
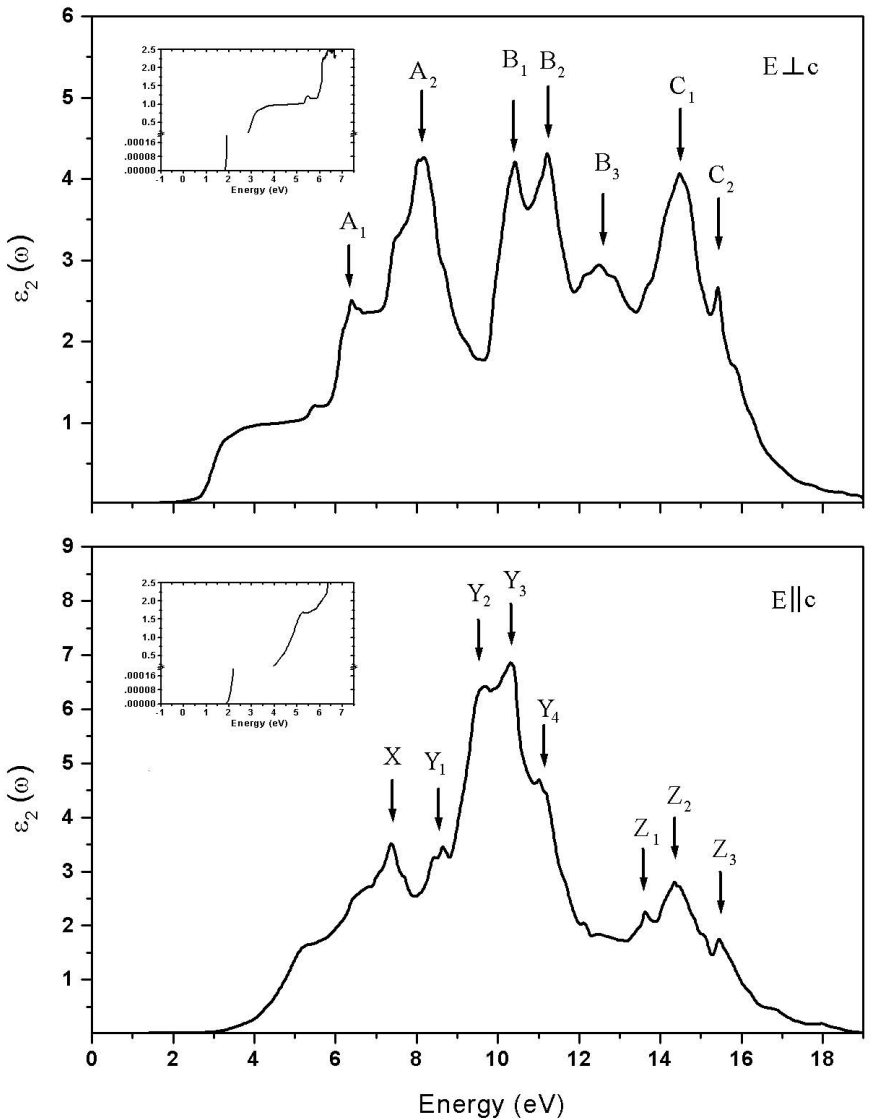

Fig. 3. The imaginary part of the dielectric function for polarization vectors perpendicular $\mathbf{E} \perp$ and parallel $\mathbf{E} \|$ to the $c$ axis. The labels are chosen arbitrary at critical points. The onsets are included to indicate the fundamental absorption edge.

the bottom of the conduction band along $\mathrm{X}-\mathrm{M}$ direction. Near the prominent feature $Y_{3}$, located at $10.29 \mathrm{eV}$, we find three additional features. The first one appears at $8.62 \mathrm{eV}$, attributed to $Y_{1}$ and resulting from transitions between valence band 11 to the second conduction band along the $\mathrm{M}-\Gamma$ direction. The second feature, labelled $Y_{2}$, occurs at $9.60 \mathrm{eV}$ and results from transitions between valence bands $13-14$ to the conduction bands $4-5$ in the neighborhood of the $\mathrm{X}-\mathrm{M}$ direction. The third feature, labelled $Y_{4}$, occurs at $11.04 \mathrm{eV}$, and results from transition between valence band 13 to the third conduction band. Three other features on the high-energy side appear as shoulders at $13.62,14.36$ and $15.42 \mathrm{eV}$. The first transition between valence band 15 and conduction band 8 , located in the neighborhood of $\Gamma-\mathrm{X}$ direction, is associated with peak $Z_{1}$. The next major peak is a result of transitions between valence bands $13-14$ and conduction bands 7 location in the neighborhood of the $\mathrm{R}-\Gamma$ direction and is associated with peak $Z_{2}$. The last peak labelled $Z_{3}$ results from transition between valence bands 13 to conduction band 9 in the neighborhood of the $\mathrm{R}-\Gamma$ direction.

\section{Conclusion}

We have used the new FP-LAPW method, within the LDA for exchange-correlation potential, to investigate the 
band structure and the optical properties of $\mathrm{GeO}_{2}$ in the rutile structure. The geometry of rutile-type structure of $\mathrm{GeO}_{2}$ has been optimized and good agreement is obtained with experiment. The fundamental band gap of the rutile $\mathrm{GeO}_{2}$ is direct at the center of the Brillouin zone. From the imaginary part of the dielectric function, we have studied the different optical transitions, in which we have presented the different peak structures associated with their assignment of the direct interband transitions. Furthermore, we are able to account for differences in electronic polarizations for the dielectric function calculation.

This work was supported by the Swiss National Science Foundation and the Swiss State Secretariat for Education and Science.

\section{References}

1. J. Muller, H.R. Blank, J. Am. Chem. Soc. 46, 2338 (1924)

2. A.W. Laubengauer, D.S. Morton, J. Am. Chem. Soc. 54, 2303 (1932)

3. W.H. Zachariasen, Z. Kirist. 67, 226 (1928)

4. T.G. Kujumzelis, Z. Phys. 100, 221 (1935)

5. R.C. Liebermann, Phys. Earth Planet. Inter. 7, 461 (1973)

6. R. Hazen, L.M. Finger, J. Phys. Chem. Solids 42, 143 (1981)

7. A. Navrotsky, J. Inorg. Nucl. Chem. 33, 1119 (1971)

8. K.V.K. Rao, S.V.N. Naidu, L. Iyengar, J. Am. Ceram. Soc. 51, 467 (1968)

9. W.H. Baur, A.A. Khan, Acta Cryst. B 27, 2133 (1971)

10. I. Jackson, Phys. Earth Planet. Inter. 13, 218 (1976)

11. L. Bertini, P. Ghigna, M. Scavini, F. Cargnoni, Phys. Chem. Chem. Phys. 5, 1451 (2003)

12. D.M. Christie, J.R. Chelikowsky, Phys. Rev. B 62, 14703 (2000)
13. A. Svane, E. Antoncik, J. Phys. Chem. Solids 48, 171 (1987)

14. L.H. Jolly, B. Silvi, P. D'Arco, Eur. J. Mineral. 6, 7 (1994)

15. A.A. Camargo, J.A. Igualada, A. Beltrán, R. Llusar, E. Longo, J. Andrés, Chem. Phys. 212, 381 (1996)

16. J. Mimaki, T. Tsuchiya, T. Yamanaka, Z. Kristallogr. 215, 419 (2000)

17. M. Stapelbrock, B.D. Evans, Solid Stat. Commun. 25, 959 (1978)

18. K. Shiraki, T. Tsuchiya, S. Ono, Acta Cryst. B 59, 701 (2003)

19. H. Wang, G. Simmons, J. Geophys. Res. 78, 1262 (1973)

20. A.A. Bolzan, B. Fong, J.B. Kenedy, Ch.J. Howard, Acta Crystallogr., Sect. B: Struct. Sci. B 53, 373 (1997)

21. J.K. Burdett, Inorg. Chem. 24, 2244 (1985); J.K. Burdett, T. Hughbanks, G.J. Miller, J.W. Richardson, J.V. Smith, J. Am. Chem. Soc. 109, 3639 (1987)

22. P. Blaha, K. Schwarz, G.K.H. Madsen, D. Kvasnicka, J. Luitz, WIEN2k, An augmented plane wave plus local orbitals program for calculating crystal properties (Vienna University of Technology, Austria 2001)

23. P. Blaha, K. Schwarz, J. Luitz, Wien97, Vienna university of technology Improved and Updated Unix Version of the original copyrighted WIEN-code (1997)

24. P. Blaha, K. Schwarz, P. Sorantin, S.B. Trickey, Comput. Phys. Commun. 59, 399 (1990)

25. P.I. Sorantin, Ph.D. thesis (Technische Univesität Wien, Vienna, 1990)

26. L. Hedin, B.I. Lundqvist, I. Phys. C 4, 2064 (1971)

27. H. Ehrenreich, M.H. Cohen, Phys. Rev. 115, 786 (1959)

28. S.Y. Ren, W.A. Harrison, Phys. Rev. B 23, 762 (1981)

29. Z. Lodziana, K. Parlinski, J. Hafner, Phys. Rev. B 63 , $134106(2001)$

30. L.H. Jolly, B. Silvi, P. D'Arco, Eur. J. Mineral. 6, 7 (1994) 